OPEN ACCESS

Edited and reviewed by: Leye Wang

Peking University, China

*Correspondence: Siobhán Clarke siobhan.clarke@scss.tcd.ie Ouri Wolfson wolfson@uic.edu

Specialty section:

This article was submitted to Smart Technologies and Cities, a section of the journal

Frontiers in Sustainable Cities

Received: 27 March 2020

Accepted: 05 May 2020

Published: 27 May 2020

Citation: Clarke S and Wolfson O (2020) Smart Technologies and Cities, Specialty Grand Challenge.

Front. Sustain. Cities 2:22. doi: 10.3389/frsc.2020.00022

\section{Smart Technologies and Cities, Specialty Grand Challenge}

\author{
Siobhán Clarke ${ }^{1 *}$ and Ouri Wolfson ${ }^{2 *}$ \\ ${ }^{1}$ Trinity College Dublin, Dublin, Ireland, ${ }^{2}$ Department of Computer Science, University of Illinois at Chicago, Chicago, IL, \\ United States
}

Keywords: technology, cities, grand challenge, research paper call, urban problems, urban challenges

\section{URBAN PROBLEMS}

Today, a majority ( $\sim 55 \%)$ of the world's population live in cities, with 3 million people moving to cities every week and projections for $\sim 68 \%$ of the world's population to live in cities by 2050 (United Nations, 2018). While some studies indicate that living standards can be better in urban areas compared to villages (Gollin et al., 2017), there are significant societal challenges that are exacerbated by large population growth in urban areas that have constrained resources, such as road networks, public transport, energy, water, waste management, healthcare, and so on. The world's population is aging, with the $60+$ year old population growing from $12.5 \%$ in 2015 to $20 \%$ by 2050 , with implications for health care, social security, family life and transport. Cities' ecological footprint is very significant, with Earth Overshoot Day becoming earlier and earlierprojected to be on July 29th 2020, the earliest ever. World premature deaths related to air pollution were estimated at 4.2 million people in 2016. The WHO surveyed 4,300+ cities and concluded that only $20 \%$ of the urban population surveyed live in areas with recommended air quality levels ${ }^{1}$. Air pollution is getting worse, with causes including increased urban power demand, rising numbers of private cars, inefficient building heating/cooling systems, and the use of solid fuels for heating and cooking. Waste management remains a huge problem, though levels of municipal waste are decreasing in many cities. Urban sprawl also remains a problem, with the effect of increasing car dependency, increasing emissions, more costly local services (e.g., water, energy), and farmland loss affecting food production and reducing biodiversity. Increasing car dependency is likely to increase congestion and transport costs, and reduce quality of life. Population density in urban areas also introduces problems of infectious disease control, as the recent Covid-19 epidemic has demonstrated. These problems will be increasingly compounded by climate change, particularly in emergencies such as floods, fires, hurricanes, and tornadoes that are becoming more frequent and more intense. Interdependencies among food, water, transportation, energy, and health make such emergencies more dangerous for cities, and more stressful for municipalities in terms of preparedness.

Furthermore, climate change is a challenge even in non-emergency situations. For example, sea level rise and human migration are growing problems that will need continuous monitoring and management. Other challenges include increasing inequality and aging infrastructure. A complicating factor is that the above challenges need to be addressed while producing and maintaining economic prosperity; not only that, but solutions also need to consider equity of disadvantaged groups and minorities.

\footnotetext{
${ }^{1}$ World Health Organization. Urban Health and Sustainable Development.
} 


\section{TECHNOLOGIES}

It is clear that multiple disciplines and stakeholders need to collaborate to address the very real challenges our cities face now and in the future. Reducing our focus to technology, there have been significant advances in various technologies that make us optimistic that technology can be a force for enabling exciting solutions to cities' challenges. We highlight four of those technologies here.

First, it is currently technically possible to have real-time gathering of big data that models the environment at a very fine level of detail. This capability is due to significant advances in sensing technologies and crowd-sourcing. Real-time data can be gathered from a myriad of sources, located in a myriad of places. Sources include citizens engaging in crowdsourcing activities, video feeds from cameras dotted around the city, wireless sensors that detect temperature, noise or pollution levels, traffic, etc. Indeed, most human activities and natural events can be automatically sensed, providing a real-time model of the urban environment. Places where such data gathering devices may be located include static places such as lamp-posts, bus-shelters, building roofs or mobile places such as e-bicycles, trams, buses, taxis, and citizens. The availability of such real-time data makes it much more viable to make sensible decisions about what changes should be made to how the city is working. There's a flood on certain streets? Re-route the traffic. There's a water leak in an important pipe in the city? Turn off surrounding pipes. There's an unexpected event resulting in a build-up of the number of citizens that need to leave an area? Assign more buses. Need to identify the contacts of each infected person to control the spread of infectious diseases such as Covid-19? Track people via their smartphones. There are countless examples of where knowing the right information at the right place at the right time could be helpful.

Second, communications capabilities worldwide have sky-rocketed. Estimates suggest that two-thirds of the world's population own a mobile device and over half the world's population access the Internet. Short-range communications such as Bluetooth and Zigbee are hugely popular, and enable localized information transfer. Cellular networks have advanced to $5 \mathrm{G}$, which has the potential to very significantly increase capacity and reduce latency, enabling sharing of jitter-free video and $\mathrm{AR} / \mathrm{VR}$ information and allows the network to remain resilient to the addition of billions of Internet of Things devices. Good quality-of-service for low-latency, high bandwidth information flow is essential to take full advantage of the information available in real-time. Wirelessly connected vehicles (vehicle-to-vehicle, vehicle-to-infrastructure, and vehicle-to-device) have the potential to increase safety, mobility, and convenience.

Third, artificial intelligence techniques encompassing technologies for data analysis, event prediction, relevance feedback, and machine learning are evolving to the point where it enhances real-time decision-making that improves city dynamics and operations. Furthermore, the Robotics branch of $\mathrm{AI}$, and particularly autonomous vehicles, have the potential to address many of the urban transportation problems, but unless carefully analyzed and managed, they can increase congestion and pollution.

Finally, novel transportation technologies such as electric vehicles, shared mobility, on-demand ride-hailing (Uber, Lyft, Didi), micro-mobility (bicycles and scooters) are emerging trends that have the potential to address urban environmental problems and congestion (Sperling, 2018). However, these technologies need to be optimized. Some examples include route planning to address the limited range of electric vehicles [Sperling] and optimization of dynamic ride-sharing (Agatz et al., 2012).

\section{CHALLENGES}

Of course, while there are very exciting technological advances, there are also significant challenges to realizing the full benefits of those advances. Here we introduce some of these challenges. First, the adoption of smart technologies in cities has the potential to constitute a very high threat to the security and privacy of citizens. ACM Transactions on Privacy and Security is a scientific journal dedicated to these issues. However, interesting questions remain open, such as how to identify and counter emerging threats in cities that arise from changes to the digital, physical, and social spaces; or how do we understand the implications of technologies, and implement security and privacy controls in a digital environment where automated changes are made on the environment in response to real-time knowledge gathering/analysis; or how do we manage the trade-offs between security and privacy requirements and other service qualities such as efficiency, usability, governance, and human behavior?

In addition, while we anticipate further innovation along many dimensions of technology that could be of benefit to the management of cities, the interoperability of these heterogeneous technologies from multiple competing vendors remains a challenge. Of course there is a lot of existing work on data fusion, integration, and mining (Bleiholder and Naumann, 2008; Atluri et al., 2018). Standards bodies have made significant progress in many areas, but technology innovation seems to constantly out-run standards, and cities are in need of solutions urgently. Heterogeneity is present in the technologies underpinning data sources, data representation, data analysis, communications, automated digital agent objectives, security/privacy techniques, and many more. For each of these individually, and their interoperability, open research questions remain on how to extract meaningful and appropriate data, how to communicate that data and the decisions emerging from knowledge gleaned from that data, and how to deploy actionable computational models safely.

It is also not reasonable to consider smart technologies for cities without considering the citizens that live in those cities. As smart systems require the input of citizens if they are to be relevant, responsive, innovative, and sustainable, open research questions remain relating to the engagement and modeling of the role that citizens play as data providers, data consumers, and beneficiaries of innovations. Citizens' participation is required 
to determine how the city and community is managed, and for continued co-design of innovations that will have a direct impact on them.

Indeed, while artificial intelligence can hugely contribute to real-time decision making, which can result in timely interventions in the way a city is managed, real challenges emerge as to how we can ensure the ethics of the codified principles embedded in the AI system, and the equity of any resulting interventions. For example, in the Covid-19 crisis, an important question is whether it would be a good idea to allow artificial intelligence to select the people with Corona virus to be admitted to limited intensive care beds? In a recent survey, $\mathrm{Yu}$ et al. looked at current techniques for building ethics into artificial intelligence and made some recommendations for research directions related to finding better ways for accurately reporting/capturing individual biases, how to determine responsibility when things go wrong, how to monitor/enforce relevant regulations, and finding an appropriate level of transparency in decision making (Yu et al., 2018).

Finally, there are many elements of working with data that are challenging. For example, the limited availability of historical datasets and limited access to proprietary datasets, means that experimenting with predictive models and learning techniques is also limited. In addition, open challenges related to, for example, heterogeneity along multiple dimensions (vendor, format, etc.), inconsistent geographic spread of data collection, incompleteness of the collected data, and inconsistency between datasets remain.

In view of continuous advances in sensing, communication, $\mathrm{AI}$, privacy/security, etc., we feel that it is necessary to research

\section{REFERENCES}

Agatz, N., Erera, A., Savelsbergh, M., and Wang, X. (2012). Optimization for dynamic ride-sharing: a review. Eur. J. Oper. Res. 223, 295-303. doi: 10.1016/j.ejor.2012.05.028

Atluri, G., Karpatne, A., and Kumar, V. (2018). Spatio-temporal data mining: a survey of problems and methods. ACM Comput. Surv. 51, 1-41. doi: $10.1145 / 3161602$

Ayala, D., Wolfson, O., Dasgupta, B., Lin, J., and Xu, B. (2018). Spatio-temporal matching for urban transportation applications. ACM Trans. Spatial Algor. Syst. 3, 1-39. doi: 10.1145/3183344

Bleiholder, J., and Naumann, F. (2008). Data fusion. ACM Comput. Surv. 41, 1-37. doi: $10.1145 / 1456650.1456651$

Gollin, D., Kirchberger, M., and Lagakos, D. (2017). In Search of a Spatial Equilibrium in the Developing World. National Bureau of Economic Research.

Lin, J., Szczurek, P., Wolfson, O., and Xu, B. (2015). The observe-driver-and-learn platform for relevance estimation in safety warning applications from vehicular ad-hoc network. J. Transp. Res. Rec.Board 2489, 49-56. doi: 10.3141/2489-06

Palade, A., and Clarke, S. (2020). Collaborative agent communities for resilient service composition in mobile environments. IEEE Trans. Serv. Comput. doi: 10.1109/TSC.2020.2964753. [Epub ahead of print]. the way all smart technology advances impact cities; and how the advances need to be built upon for the benefit of society in cities. Work on urban computing (Zheng, 2018; White and Clarke, 2020) addresses these adaptations, but much more remains to be done.

Furthermore, the technologies and needs are continuously evolving, and there is therefore an increasing number of possibilities for their innovative combinations. For example, crowd-sourcing, mobile sensing, and algorithmic game theory can be combined to address resource discovery in urban transportation (Ayala et al., 2018), or artificial intelligence and edge computing can be combined to increase the resilience of service provisioning in urban environments (Palade and Clarke, 2020). Similarly, advances in AI and vehicular ad-hoc networks can be combined to enable safer mobility (Lin et al., 2015).

In this new section of Frontiers in Sustainable Cities, we invite papers that report on innovative research results that illustrate the design, implementation, and validation of smart technologies that address city management challenges.

\section{AUTHOR CONTRIBUTIONS}

All authors listed have made a substantial, direct and intellectual contribution to the work, and approved it for publication.

\section{FUNDING}

This work was partially supported by Science Foundation Ireland under grant number 16/SP/3804 (to SC) and NSF grant IIP1534138 and UIC grant MY-AIR (to OW).

Sperling, D. (2018). Three Revolutions: Steering Automated, Shared, and Electric Vehicles to a Better Future. Island Press.

United Nations (2018). Revision of World Urbanization Prospects.

White, G., and Clarke, S. (2020). Urban intelligence with deep edges. IEEE Access. doi: 10.1109/ACCESS.2020.2963912. [Epub ahead of print].

Yu, H., Shen, Z., Miao, C., Leung, C., Lesser, V. R., Yang, Q. (2018). "Building ethics into artificial intelligence," in Proceedings of the 27th International Joint Conference on Artificial Intelligence (IJCAI'18) (Stockholm).

Zheng, Y. (2018). Urban Computing. Cambridge, MA: MIT Press.

Conflict of Interest: The authors declare that the research was conducted in the absence of any commercial or financial relationships that could be construed as a potential conflict of interest.

Copyright (c) 2020 Clarke and Wolfson. This is an open-access article distributed under the terms of the Creative Commons Attribution License (CC BY). The use, distribution or reproduction in other forums is permitted, provided the original author(s) and the copyright owner(s) are credited and that the original publication in this journal is cited, in accordance with accepted academic practice. No use, distribution or reproduction is permitted which does not comply with these terms. 